Review Article

\title{
Melanoma Immunotherapy: Mechanisms and Opportunities
}

\author{
Yu Xu ${ }^{1,3}$, Anita Van Der Sande ${ }^{1}$ Jennifer A. Lo ${ }^{1}$, David E. Fisher ${ }^{1,2^{*}}$ \\ ${ }^{1}$ Cutaneous Biology Research Center, Massachusetts General Hospital, Charlestown, Massachusetts, USA \\ ${ }^{2}$ Department of Dermatology, Massachusetts General Hospital, Boston, Massachusetts, USA \\ ${ }^{3}$ Department of Biomedical Engineering, Johns Hopkins University, Baltimore, Maryland, USA
}

*Corresponding Author: David E. Fisher, MD, PhD, Department of Dermatology, East Cutaneous Biology Research Center, Massachusetts General Hospital, Building 149, $3^{\text {rd }}$ Floor, $13^{\text {th }}$ Street Charlestown, MA 02129, USA. Tel: (+1) 617-643-5428; E-mail: dfisher3@partners.org

\begin{abstract}
Immune checkpoint blockade via inhibition of Cytotoxic T Lymphocyte Antigen 4 (CTLA-4) and Programmed Cell Death 1 Receptor (PD-1) has demonstrated significant clinical benefits in treating melanoma and other types of cancers and has since become a very progressive field in cancer research. Despite durable tumor regression observed in some patients, response rates to CTLA-4 and PD-1 still have room for improvement. There are many additional immune modulatory pathways, including inhibitory molecules expressed on tumor cells and secretion of pro-inflammatory cytokines by lymphatic cells that could potentially be targeted to enhance the anti-tumor responses to PD-1 and CTLA-4. Here, we review the current status of CTLA-4 and PD-1 inhibitors in the treatment of melanoma and several therapeutic targets and strategies that may synergize with checkpoint blockades.
\end{abstract}

Received date: September 29, 2015

Accepted date: November 20, 2015 Published date: November 26, 2015

Citation: Fisher, D.E., et al. Melanoma Immunotherapy: Mechanisms and Opportunities. (2015) Invest Dermatol Venereol Res 1(2): 33-39.

DOI: $10.15436 / 2381-0858.15 .010$

\section{Introduction}

Melanoma is one of the most lethal and aggressive forms of skin cancer and has historically been a treatment-resistant malignancy. However, in recent years improved understanding of the molecular nature of melanoma and the interaction of melanoma cells with the immune system has resulted in multiple new immunotherapeutic strategies, of which immune checkpoint inhibitors are the most advanced ${ }^{[1]}$.

Immunotherapy aims to generate or enhance T-cell responses against tumor cells. Activation of naive T-cells requires two signals: T-cell Receptor (TCR) signaling and co-stimulatory signaling (Figure 1). The first signal is provided by binding of an antigenic peptide/major Histocompatibility complex on the surface of an Antigen-Presenting Cell (APC) to the T-cell receptor. Melanoma is characterized by a high rate of ultraviolet induced mutations. These mutations lead to changes in protein coding sequence, which the immune system recognizes as altered proteins epitopes, also known as neoantigens ${ }^{[2,3]}$.

A second co-stimulatory interaction between the T-cell and APC can modulate TCR signaling positively or negatively. These co-stimulatory and co-inhibitory molecules, expressed on the surface of the T-cell, mediate immune checkpoints ${ }^{[4]}$. Under normal physiological conditions, immune checkpoint pathways maintain self-tolerance and limit collateral tissue damage during anti-microbial immune responses. Cancer can exploit inhibitory immune checkpoints to evade tumor destruction.

The most clinically advanced checkpoint inhibitors to date are blocking monoclonal antibodies that target the CTLA-4 and PD-1 pathways. CTLA-4 is a Transmembrane protein on T-cells that competes with another receptor on T-cells, CD28, for the same ligands. These ligands, CD80 (also known as B7.1) and CD86 (also known as B7.2) are expressed on APCs. Binding between CD80/86 and CD28 stimulates T-cell activation, while binding to CTLA-4 inhibits T-cell activation (Figure 1) ${ }^{[5,6]}$. Therefore, the expression level of CTLA-4 dictates the threshold of T-cell activation by APCs, and CTLA-4 inhibitors promote T-cell activation by blocking the binding between CTLA-4 and B7. Like CTLA-4, PD-1 is a co-inhibitory surface receptor expressed on T-cells. The PD-1 ligands PD-L1 and PD-L2 are expressed in a wide variety of peripheral tissues, including tumors. Upon binding to its ligands, PD-1 negatively regulates the function of activated T-cells. PD-L1 expression in the tumor microenvironment is one important mechanism of tumor-mediated immune evasion (Figure 1) ${ }^{[7,8]}$. PD-1 inhibitors block the binding between PD-1 and PD-L1/PD-L2, whereas PD-L1 inhibitors more selectively block the interactions between PD-L1 and PD-1/CD80. Both prevent T-cell exhaustion 


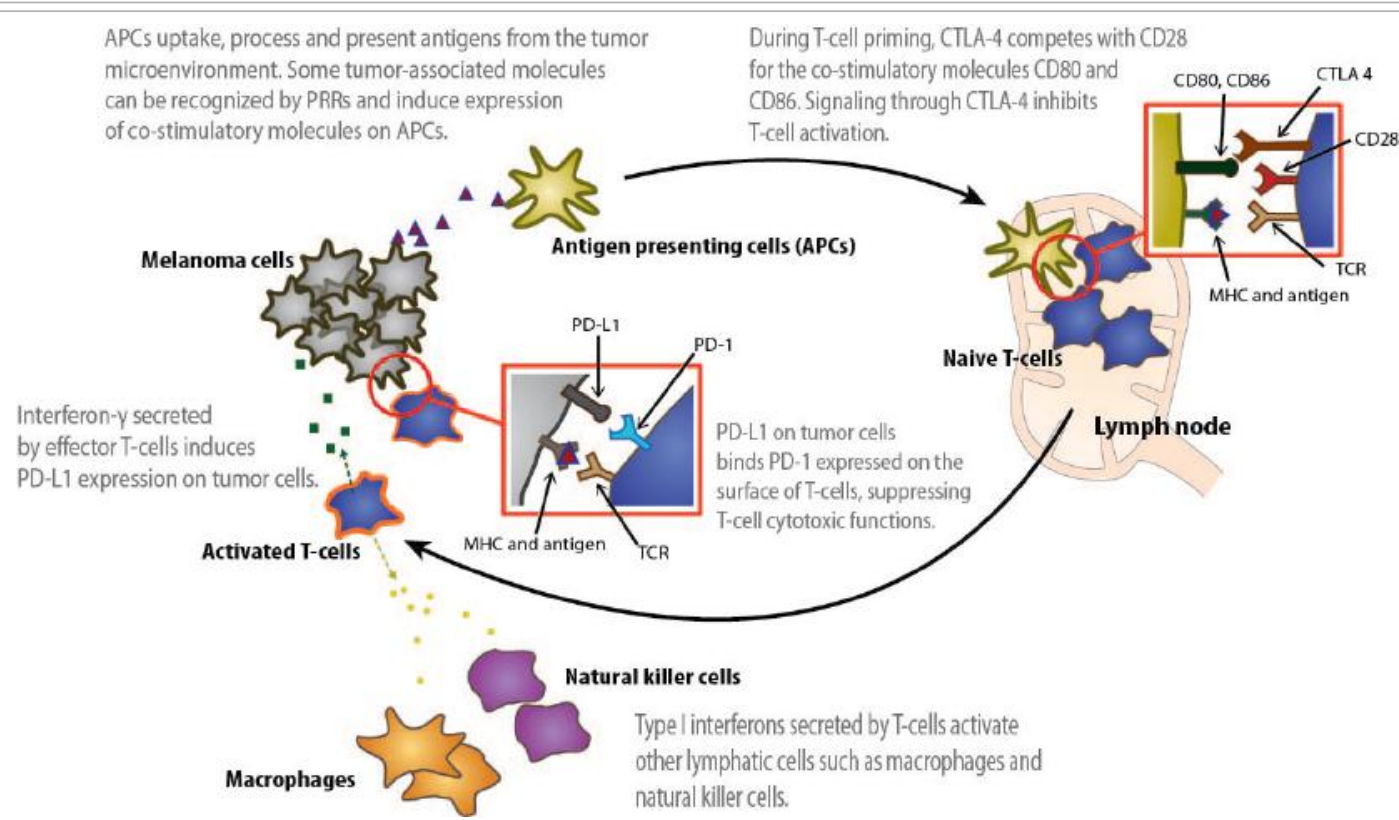

Figure 1: Anti-tumor immunity. The immune response against melanoma begins with the uptake of tumor-associated antigens by APCs, which then migrate to lymphatic tissues to activate naive T-cells. Two signals are required for T-cell activation: antigen presentation and co-stimulatory signaling. In this process, CTLA-4 competes with the co-stimulatory molecule CD28 for ligands CD80 and CD86, inhibiting naive T-cell activation. Blocking monoclonal antibodies against CTLA-4 prevent binding of CTLA-4 to its ligands, allowing T-cell activation. Activated T-cells secrete type I interferons that can activate other immune cells including natural killer cells and macrophages. Effector T-cells subsequently traffic to and infiltrate tumors, destroying cancer cells. During this stage, PD-L1 expressed on tumor and stromal cells bind PD-1 receptors on T-cells. PD-1 signaling inhibits T-cells. In addition, interferon- $\gamma$ secreted by tumor infiltrating lymphocytes upregulates PD-L1 expression on tumor cells, suppressing T-cell responses. PD-1 inhibitors reinvigorate exhausted T-cells by blocking the interaction between PD-L1 and PD-1.

and PD-1 and PD-L1 inhibitors have demonstrated comparable clinical efficacy ${ }^{[9]}$. In summary, CTLA-4 primarily suppresses the activation of naive T-cells by APCs while PD-1 inhibits previously-activated effector T-cells ${ }^{[10,11]}$. This is thought to explain why PD-1 inhibitors have a lower toxicity profile compared to CTLA-4 inhibitors. Although CTLA-4 and PD-1 inhibitors are currently the most promising cancer immunotherapy treatment options, most melanoma patients still do not respond to these therapies. In this review, we discuss progress in the use of CTLA-4 and PD-1 inhibitors for the treatment of melanoma, and different mechanisms and therapeutic methods that could potentially improve the efficacy of CTLA-4 and PD-1 inhibitors.

\section{Current Status of CTLA-4 and PD-1 Inhibitors}

There are three checkpoint blockade agents that have been approved by the FDA for the treatment of advanced melanoma: Ipilimumab (antibody against CTLA-4), Pembrolizumab and Nivolumab (antibodies against PD-1). Compared to traditional targeted therapies, such as BRAF inhibitors, checkpoint blockade offers more durable responses but with lower response rates. A recently published meta-analysis of survival data following Ipilimumab therapy reported that Ipilimumab extended overall survival from approximately 8 months to 11.4 months in patients with metastatic melanoma. A plateau survival rate of $21 \%$ was reached around year 3 , with follow-up of up to 10 years $^{[12]}$.

Compared to Ipilimumab, PD-1 inhibitors elicit higher response rates with fewer side effects ${ }^{[13,14]}$. In a recently published phase III study comparing Pembrolizumab versus Ipilimumab in the treatment of advanced melanoma ${ }^{[13]}$, the estimated 12-month survival rates were $74.1 \%$ for Pembrolizumab administered every 2 weeks at the dose of $10 \mathrm{mg} / \mathrm{kg}, 68.4 \%$ for Pembrolizumab administered every 3 weeks at the dose of $10 \mathrm{mg} / \mathrm{kg}$, and $58.2 \%$ for Ipilimumab administered every 3 weeks at the dose of $3 \mathrm{mg} /$ $\mathrm{kg}$. The response rates for Pembrolizumab administered every 2 weeks and 3 weeks were $33.7 \%$ and $32.9 \%$ respectively, significantly higher than the response rate for Ipilimumab administered every 3 weeks (11.9\%). Rates of grade 3 - 5 adverse events in the two Pembrolizumab groups $(13.3 \%$ in the 2 -week group and $10.1 \%$ in the 3 -week group) were lower than the rate in the Ipilimumab group (19.9\%). It should be noted that clinical trials for Pembrolizumab and Nivolumab started 6 years later than the trials for Ipilimumab and clinical studies with longer follow-up are needed to fully evaluate the efficacy of PD-1 inhibitors.

The non-redundant mechanisms of CTLA-4 and PD-1 provide a rationale for combination of CTLA-4 and PD-1 inhibitors. Several clinical studies have shown superior response rates and progression free survival with combined Ipilimumab and Nivolumab treatment ${ }^{[15-17]}$. For example, the phase III study testing Nivolumab and Ipilimumab combined therapy in patients with metastatic melanoma reported an objective response rate of $43.7 \%$ in the Nivolumab group $(3 \mathrm{mg} / \mathrm{kg}$ administered every 2 weeks), $19.0 \%$ in Ipilimumab group $(3 \mathrm{mg} / \mathrm{kg}$ administered every 3 weeks) and $53.6 \%$ in the combined Nivolumab Ipilimumab group ( 4 doses of $1 \mathrm{mg} / \mathrm{kg}$ Nivolumab plus $3 \mathrm{mg} / \mathrm{kg}$ Ipilimumab administered every 3 weeks, followed by $3 \mathrm{mg} / \mathrm{kg}$ Nivolumab administered every two weeks) ${ }^{[17]}$. The median progression-free survival for the combined therapy was 11.5 months, compared to 2.9 months for Ipilimumab, and 6.9 months for Nivolumab. It should be noted that in patients with high PD-L1 expression on tumor cells, the median progression-free survival was the same in the combined therapy group and in the Nivolumab group (14 months), but in patients with tumors that expressed a low level of PD-L1, progression-free survival was longer in the combined therapy group (11.2 months vs. 5.3 months). This suggests that PD-L1 expression in the tumor is associated with but does not 
perfectly predict PD-1 inhibitor efficacy. Grade 3 or 4 adverse events (grade 3 or 4 ) were much more frequent in the combined therapy group than in the two monotherapy groups $(55.0 \%$ in the combined group, $16.3 \%$ in the Nivolumab group and $27.3 \%$ in the Ipilimumab group). Higher rates of adverse events resulting from the combined CTLA-4 and PD-1 therapies were observed in all studies, raising safety concerns regarding combined therapy ${ }^{[15-18]}$.

\section{Mechanisms and Therapeutic Strategies to Enhance Im- mune Checkpoint Blockade Efficacy}

The potential for highly durable responses has led to great interest in developing synergistic combinatorial approaches with other treatment modalities that could expand the proportion of responders to checkpoint blockade.

\section{Overview of anti-tumor immune response}

The immune response against tumor cells can be conceptualized in four steps (Figure 1): 1) APCs are activated by tumor-associated antigens and present antigens to T-cells in the lymphatic system; 2) APCs activate antigen-specific T-cells; 3) activated T-cells traffic and infiltrate into the tumor; 4) Cytotoxic T-cells recognize and attack cancer cells ${ }^{[19]}$. In this process, CTLA-4 and PD-1 inhibitors are known to enhance T-cell activation and Subsequent cancer cell recognition and killing ${ }^{[20]}$, but APC activation and T-cell trafficking must be addressed by other therapeutic methods.

\section{Role of the inflammatory tumor microenvironment in im- munotherapy}

Density of tumor infiltrating cytotoxic CD8+ T-cells is one of the best predictors of response to current checkpoint blockade therapies ${ }^{[21-23]}$. Characterization of the tumor microenvironment reveals two immunologic phenotypes: inflamed and non-inflamed tumors ${ }^{[24]}$. Many studies have shown that inflamed tumors, with dense T-cell infiltration and high concentrations of type I interferons, are more likely to respond to checkpoint blockade. Non-inflamed tumors lack T-cell infiltrate and may require additional interventions to achieve optimal inflammation and innate immune activation in the tumor microenvironment. However, the role of the inflammation is complex. Previous studies have shown a higher incidence of cancer in tissues that have experienced chronic inflammation, suggesting a pro-tumorigenic effect in some inflammatory contexts ${ }^{[25-28]}$. Chronic inflammation in the tumor microenvironment may contribute to tumor growth by promoting angiogenesis, cancer cell proliferation, tissue invasion and metastasis ${ }^{[29]}$. Thus, there is a delicate balance between pro and anti-tumor immunity that is determined by the relative activation of different cell types and expression of various immune mediators in the tumor microenvironment ${ }^{[25,30,31]}$. Further studies are needed to improve our understanding of optimal targets and mechanisms in the inflammatory tumor microenvironment in order to improve immunotherapy.

\section{Innate immunity is critical in the anti-tumor immune response}

Innate immunity represents the first line of immune defense. One major part of the innate immune system that is important for anti-tumor immune responses is the activation of antigen-specific T-cells by APCs. As discussed earlier, T-cell priming requires two signals: the interactions between antigens and the T-cell receptors, and the binding of co-stimulatory molecules to their receptors. The expression of co-stimulatory molecules is canonically triggered by recognition of Pathogen Associated Molecular Patterns (PAMP) by APCs. PAMPs are molecules that indicate the presence of pathogens like bacteria and viruses, and APCs detect PAMPs through Pattern Recognition Receptors (PRRs). PRRs then activate multiple pathways, and one of them leads to the production and surface expression of co-stimulatory molecules. PRRs can also stimulate secretion of type I interferons, which can induce co-stimulatory molecule expression on other cells including macrophages. It has been observed in T-cell infiltrated tumors that some pathways related to innate response are activated and have high expression of type I interferons ${ }^{[33-34]}$. In addition, a recent study reported that longterm clinical benefit following Ipilimumab therapy is associated with a provocative neoantigen signature composed of neoantigen tetrapeptides similar to viral and bacterial antigens ${ }^{[35]}$. An important question is whether therapeutic methods that could trigger these innate immune related pathways can produce additive or synergistic effects when combined with checkpoint blockade.

\section{Toll-Like Receptor activation induces dendritic cell activa- tion and maturation}

Toll-Like Receptors (TLRs) are a class of PRRs that recognize a variety of PAMPs. There are 10 types of TLRs in humans. Here, we focus on TLR-7, the receptor activated by the drug Imiquimod. Binding between TLR-7 on dendritic cells and tumor-associated nucleotide molecules up regulates the production of transcription factor Nuclear Factor-Kappa B (NF- $\mathrm{B}$ ) in a process mediated by Myeloid Differentiation Primary Response Gene (MyD88). NF- $\mathrm{B}$ then translocates into the nucleus and promotes transcription of pro-inflammatory cytokines (including Tumor Necrosis Factor Alpha (TNF- $\alpha$ ) and various interleukins) and Chemokines (including CCL2, CCL3 and CCL4). In addition, activation of TLRs also induces IFN- $\alpha$ production by $\mathrm{DCs}^{[36-38]}$. It has also been shown that stimulation of TLR7 enhances DC survival, which is characterized by increased expression of CCR7 and co-stimulatory markers, CD80 and CD86 ${ }^{[36]}$. Toll-Like receptor agonist (Imiquimod)

Imiquimod is a synthetic TLR-7 agonist that has been approved by the FDA for primary superficial basal cell carcinoma. Imiquimod Monotherapy has limited efficacy in treating metastatic melanoma ${ }^{[39]}$. Combined Imiquimod and melanoma peptide vaccine therapy has been tested in Murine models, and the combined therapy demonstrated measurable anti-tumor immunity ${ }^{[40]}$. Vaccine and Imiquimod combined therapy is currently under investigation.

\section{STING activation promotes antigen presentation and T-cell priming}

The Stimulator of Interferon Genes Complex (STING) is an endoplasmic reticulum resident protein that functions as an adaptor molecule for cytosolic DNA recognition. The STING pathway is essential for tumor-associated antigen uptake by APCs and T-cell priming ${ }^{[41]}$. Tumor-derived DNA is taken up by host APCs, which generate cyclic dinucleotides through enzyme Cyclic-GMP-AMP Synthase (cGAS) ${ }^{[42-44]}$. Binding of cyclic dinucleotides to STING leads to Phosphorylation of Interferon 
Regulatory Factor 3 (IRF3), which in turn promotes transcription and production of type I interferons by APCs, which are important for early innate immune responses (Figure 2) ) $^{[3,45,46]}$.

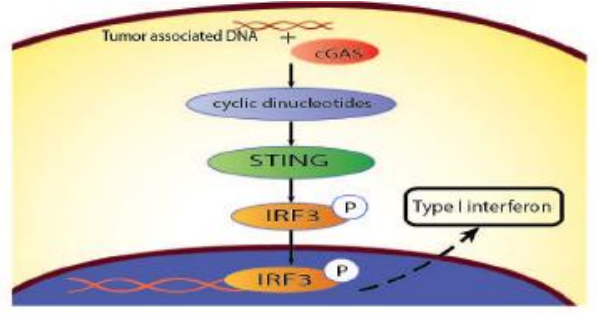

Figure 2: The STING signaling pathway. After up-take of tumor-associated DNA, host APCs generate cyclic dinucleotides in a process mediated by enzyme cGAS. Cyclic dinucleotides directly activate STING, which specifies phosphorylation of IRF3 by the kinase TBK1. Phosphorylated IRF3 moves into the nucleus to enhance transcription of type I interferons, which play a critical role in early innate immune response.

\section{STING agonist}

The finding that the STING pathway can induce T-cell infiltration provides the basis for using STING agonists as a cancer therapeutic strategy. Mixed Linkage-RR-S2 CDA (MLRR-S2 CDA), A Cyclic Dinucleotide (CDN) derivative and STING agonist has shown activity in mouse models. Following intra tumoral injection of ML-RR-S2 CDA in one study, mice had significant and durable tumor regressions, and complete responders showed immune resistance to the same cancer cell line upon rechallenge ${ }^{[47]}$. CDNs are promising candidates for study in clinical trials.

\section{Activated $\beta$-Catenin signaling pathway impairs dendritic cell activation and T-cell proliferation}

$\beta$-catenin is a key intracellular signaling transducer, and dysregulation of $\beta$-catenin has been linked to many physiological phenomena, including the development of cancer and the exclusion of T-cell infiltration into tumors. An interesting observation is that $\beta$-catenin signaling is activated in almost half of all non-inflamed melanomas ${ }^{[48]}$. Recent studies have shown that activated $\beta$-catenin signaling in tumor cells causes immune suppression mainly via blocking dendritic cell recruitment and subsequent T-cell activation.

It has been demonstrated in murine models that $\beta$-catenin induces the expression of a transcriptional repressor, AMP-Dependent Transcription Factor 3 (ATF3), which suppresses Chemokine Ligand 4 (CCL4) Production. CCL4 is a key Chemokine in dendritic cell recruitment, and a low level of CCL4 results in impaired dendritic cell activation. The efficacy of checkpoint blockade is greatly reduced in $\beta$-catenin stabilized mouse tumor models, suggesting that the $\beta$-catenin pathway could be a potential therapeutic target in combination with checkpoint blockade ${ }^{[48]}$.

It has also been shown in human melanoma cells that nuclear $\beta$-catenin directly binds to the IL-10 promoter and increases IL-10 transcription and secretion. IL-10 induces differentiation of immature dendritic cells into regulatory dendritic cells, which impairs ability of T-cells to secrete IFN- $\gamma$ and cytotoxins such as granzyme ${ }^{[49]}$. This suggests that inhibiting $\beta$-catenin accumulation might not only promote dendritic cell and T-cell activation, but also improve T-cell function.

\section{$\beta$-Catenin inhibitor}

Although $\beta$-catenin has long been identified as a potential therapeutic target, the clinical development of $\beta$-catenin inhibitors is still at an early stage. Currently, several anti $\beta$-catenin agents have been reported in pre-clinical studies ${ }^{[0,51]}$, none in combination with immune checkpoint inhibitors.

\section{Inhibition of the MAPK pathway enhances cancer cell recog- nition by $T$-cells and augments T-cell function}

The BRAF (V600E) mutation, the most common mutation in melanoma, results in constitutive activation of the Mitogen-Activated Protein Kinase (MAPK) signaling pathway. The MAPK pathway regulates cell cycle progression and cell growth, and MAPK activation is implicated in the development of many cancers. In patients with melanoma, high response rates and rapid disease stabilization or regression are observed following administration of targeted therapies that inhibit BRAF and MEK. However, early clinical benefit is followed by emergence of drug resistance in most patients. Interestingly, it has been observed in several studies that BRAF inhibitor enhances T-cell infiltration $^{[52,53]}$. BRAF inhibition also leads to up regulation of Melanocyte Differentiation Antigens (MDA) that might improve recognition of tumor cells by MDA-specific T-cells ${ }^{[53-55]}$.

MAPK pathway activation results in increased activity of the transcriptional factor C-Jun, which up regulates the transcription of PD-L1 through a process mediated by its co-factor Signal Transducer And Activator Of Transcription 3 (STAT-3) ${ }^{[56]}$. It has also been demonstrated that Oncogenic BRAF mutations enhance Interleukin-1 production. IL-1 up regulates expression of PD-L1, PD-L2 and COX-2 on Tumor-Associated Fibroblasts (TAFs), which in turn inhibit T-cell function and cytokine production $^{[57]}$.

A recent study reported synergy of PD-1 blockade and BRAF inhibitors in a mouse melanoma model, with dose-dependent increases in T-cell infiltration and PD-L1 expression following BRAF inhibition ${ }^{[58]}$. However, the first clinical trial of combined BRAF inhibitor (Vemurafenib) and Ipilimumab was terminated due to severe hepatotoxicity ${ }^{[59]}$. It has been proposed that the combination of checkpoint blockade with MEK inhibition may achieve similar efficacy with fewer side effects compared to BRAF inhibition. The triple combination of BRAF inhibitor, MEK inhibitor and PD-1 inhibitor has been tested in mouse models with promising results ${ }^{[60]}$. The phase I trial testing Dabrafenib, Trametinib, Ipilimumab and Nivolumab quadruple therapy is currently underway.

\section{Radiation enhances trafficking and infiltration of T-cells and tumor cell recognition}

Radiation therapy causes cancer cell death by inducing DNA damage. During the process, radiation promotes tumor antigen presentation and T-cell recruitment. Recent studies have shown that IFN- $\gamma$ plays a key role in inducing T-cell trafficking after radiation. Radiation stimulates secretion of IFN- $\gamma$, which up regulates the expression of Vascular Cell Adhesion Molecule 1 (VCAM-1) on tumor vessels, facilitating T-cell movement into the tumor ${ }^{[61,62]}$. IFN- $\gamma$ also stimulates production of C-X-C Motif Chemokine Ligand 9 (CXCL9) by tumor cells, including C-X-C Motif Chemokine Ligand 10 (CXCL10) that is known to promote T-cell infiltration ${ }^{[61]}$.

Radiation also augments T-cell recognition of melano- 
mas by expanding endogenous peptide repertoires in melanoma cells ${ }^{[63,64]}$. It has been established that radiation can increase peptide production and modify existing proteins. The proteins will then be degraded by Proteasomes, generating more tumor-associated antigens that can be presented by $\mathrm{MHC}$, promoting a more immunogenic tumor microenvironment ${ }^{[64]}$.

The phase I clinical trial testing combined Ipilimumab and radiotherapy reported partial responses in $18 \%$ and stable disease in $18 \%$ of patients with metastatic melanoma. Up regulated PD-L1 expression on tumor cells was observed, consistent with T-cell exhaustion that could explain the low efficacy of this combined therapy. Addition of PD-1 inhibitor enhanced anti-melanoma responses in mice treated with the Ipilimumab and radiotherapy combination ${ }^{[65]}$.

IDO inhibitor may augment the efficacy of PD-1 and CTLA4 by blocking additional pathways that impair T-cell proliferation

IFN- $\gamma$ secreted by infiltrating CD8 + T-cells induces expression of Indoleamine-2,3-dioxygenase (IDO) in tumor cells ${ }^{[66]}$. IDO is an important cytosolic enzyme in tryptophan catabolism and up regulation of IDO can result in tryptophan depletion. Lack of tryptophan causes cell cycle arrest in the G1 phase, preventing T-cell proliferation ${ }^{[67]}$. It has also been shown that IDO induces naïve T-cells to differentiate into CD4+ CD25+FoxP3+ Regulatory T-Cells (Tregs), which inhibit cytotoxic T-cell proliferation ${ }^{[68]}$.

\section{IDO inhibitor}

Several IDO inhibitors are currently available. 1-Methyl-Tryptophan (Indoximod), first discovered in 1991, is a tryptophan analogue. Its antitumor effects have been tested in mouse models of melanoma and other types of cancer ${ }^{[69-71]}$. Indoximod caused only slight tumor growth retardation, but a significant increase in cytotoxic T-cell to Treg ratio, indicating a more pro-inflammatory tumor microenvironment. The phase I/II clinical study of indoximod with Ipilimumab are underway. Another IDO inhibitor, INCB024360, has completed phase I testing and is currently being tested in several phase I and II combination clinical studies with Imiquimob or PD-L1/PD-1 inhibitors in different cancers including melanoma.

\section{Emerging checkpoint blockade}

In addition to the PD- 1 and CTLA-4 pathways, multiple other immune checkpoints have been described. Here we discuss two co inhibitory molecules, T-Cell Immunoglobulin Mucin 3 (TIM-3, also known as HAVCR2) and Lymphocyte Activation Gene 3 (LAG-3; also known as CD223), that are promising candidates for therapeutic inhibition to induce or enhance anti-tumor immunity.

\section{TIM-3 acts as an inhibitory molecule in T-cell response}

The early immune response depends on the activation of APCs. High Mobility Group Protein B1 (HMGB-1) is important in endocytosis of tumor-associated nucleic acids into dendritic cells. TIM-3 expressed on dendritic cells interferes with HMGB-1, preventing uptake of nucleic acids released by tumor cells and subsequent innate immune response activation ${ }^{[72]}$. Additionally, it has been suggested that TIM-3 affects T-cell responses indirectly via helper T-cells and regulatory T-cells.
TIM-3 blockade results in increased secretion of IFN- $\gamma$ from helper T-cells ${ }^{[73]}$. It has also been observed that TIM-3+ regulatory T-cells accumulate in the tumor before cytotoxic T-cell exhaustion and depletion of these regulatory T-cells improves cytotoxic T-cell functions ${ }^{[74]}$. As the majority of tumor-infiltrating T-cells often express both PD- 1 and TIM-3 ${ }^{[75]}$, it is expected that combined PD-1 and TIM-3 inhibition would generate stronger immune responses, as has been demonstrated in several murine studies $^{[75,76]}$

\section{LAG-3 directly and indirectly regulates cytotoxic T-cell ac- tivity}

LAG-3 is another important checkpoint that has been identified recently. LAG-3 is a surface protein found on many different types of cells and MHC class II is its only known ligand. LAG-3 suppresses cytotoxic T-cell activity indirectly by suppressing CD4+ helper T-cells and activating regulatory T-cells. LAG-3 on helper T-cells directly binds to MHC II on APCs, resulting inhibition of $\mathrm{CD} 4+$ helper T-cell proliferation and production of pro-inflammatory cytokines including IFN- $\gamma^{[77]}$. LAG-3 is also known to enhance regulatory T-cells function, although the exact mechanism is not fully understood ${ }^{[78]}$. LAG-3 expression is also up regulated on CD8+ cytotoxic T-cells in response to antigen stimulation, and blockade of LAG-3 increases CD8+ T-cell proliferation and secretion of IFN- $\gamma^{[79]}$.

In mouse experiments, the combination of LAG-3 inhibitor and PD-1 inhibitor suppresses tumor growth more than LAG-3 or PD-1 monotherapy ${ }^{[80]}$. A phase I study has been initiated for the treatment of solid tumors with anti-LAG-3 monoclonal antibody BMS-986016 administered alone or in combination with Nivolumab. Besides transmembrane LAG-3, there is also a Soluble Form of LAG-3 (sLAG-3). Like transmembrane LAG3, sLAG-3 also binds HMC class II molecules, however sLAG-3 has a stimulatory effect: sLAG-3 activates APCs when bind to MHC class II on the APC cell membrane ${ }^{[81]}$. sLAG-3 protein, IMP321, has been investigated as a potential therapeutic agent. IMP321 delivered some clinical benefit in several phase I clinical trials including patients with several types of cancer. However, more extensive study is required in order to fully evaluate the efficacy of IMP321 $1^{[82,83]}$.

\section{Conclusion}

CTLA-4 inhibitor and PD-1 inhibitors have already demonstrated the potential of immune checkpoint blockade based immunotherapy in treating melanoma. With a deeper understanding of the mechanisms of tumor immune evasion, including many other inhibitory pathways, we are discovering new therapeutic targets and strategies that may produce synergy with checkpoint blockade in patients for whom current therapies are ineffective.

Depending on the immune microenvironment, patients will likely require different combinations of treatments to achieve tumor remission. In addition to novel therapeutic modalities, the development of strategies to characterize the immune milieu with the potential to customize treatments for each individual should be another emphasis of future research. We expect to see continuous effort in the near future devoted to exploring new therapeutic targets and different combinations of treatment methods, to push the frontier of available treatments for patients 
with melanoma and other malignancies.

Conflict of Interest: No potential conflicts of interest were disclosed.

\section{Acknowledgements}

The authors gratefully acknowledge grant support from NIH (5P01 CA163222; 5R01 AR043369-19; R01CA150226; R21CA175907), the Dr. Miriam and Sheldon G. Adelson Medical Research Foundation, and the US-Israel Bi national Science Foundation, and members of the Fisher Laboratory for useful discussions.

\section{References}

1. Batus, M., Waheed, S., Ruby, C., et al. Optimal management of metastatic melanoma: current strategies and future directions. (2013) Am J Clin dermatol 14(3): 179-194.

2. Hodis, E., Watson, I.R., Kryukov, G.V., et al. A landscape of driver mutations in melanoma. (2012) Cell 150(2): 251-263.

3. Schumacher, T.N., Schreiber, R.D. Neoantigens in Cancer Immunotherapy. (2015) Science 348(6230): 69-74.

4. Chen, L., Flies, D.B. Molecular mechanisms of T cell co-stimulation and co-inhibition. (2013) Nat Rev Immunol 13(4): 227-242.

5. Walunas, T.L., Lenschow, D.J., Bakker, C.Y., et al. CTLA-4 Can Function as a Negative Regulator of T cell Activation. (1994) Immunity 1(5): 405-413.

6. Riley, J.L., Mao, M., Kobayashi, S., et al. Modulation of TCR-induced transcriptional profiles by ligation of CD28, ICOS, and CTLA-4 receptors. (2002) Proc Natl Acad Sci USA 99(18): 11790-11795.

7. Keir, M.E., Liang, S.C., Guleria, I.., et al. Tissue expression of PD-L1 mediates peripheral T cell tolerance. (2006) J Exp Med 203(4): 883-895.

8. Iwai, Y., Ishida, M., Tanaka, Y., et al. Involvement of PD-L1 on tumor cells in the escape from host immune system and tumor immunotherapy by PD-L1 blockade. (2002) Proc Natl Acad Sci USA 99(19): 12293-12297. 9. Topalian, S.L., Drake, C.G., Pardoll, D.M. Immune checkpoint blockade: a common denominator approach to cancer therapy. (2015) Cancer cell 27(4): 450-461.

10. Ahmadzadeh, M., Johnson, L.A., Heemskerk, B., et al. Tumor antigen-specific CD8 T cells infiltrating the tumor express high levels of PDand are functionally impaired. (2009) Blood 114(8): 1537-1544.

11. Parry, R.V., Chemnitz, J.M., Frauwirth, K.A., et al. CTLA-4 and PD-1 receptors inhibit T-cell activation by distinct mechanisms. (2005) Mol Cell Biol 25(21): 9543-9553.

12. Schadendorf, D., Hodi, F.S., Robert, C., et al. Pooled Analysis of LongTerm Survival Data from Phase II and Phase III Trials of Ipilimumab in Unresectable or Metastatic Melanoma. (2015) J Clinical Oncol 33(17) 1889-1894.

13. Robert, C., Schachter, J., Long, G.V., et al. Pembrolizumab versus Ipilimumab in Advanced Melanoma. (2015) N Eng J Med 372: 2521-2532.

14. Robert, C., Long, G.V., Brady, B., et al. Nivolumab in previously untreated melanoma without BRAF mutation. (2015) N Eng J Med 372(4): 320-330.

15. Postow, M.A., Chesney, J., Pavlick, A.C., et al. Nivolumab and ipilimumab versus ipilimumab in untreated melanoma. (2015) N Eng J Med 372(21): 2006-2017.

16. Wolchok, J.D., Kluger, H., Callahan, M.K., et al. Nivolumab plus ipilimumab in advanced melanoma. The (2013) N Eng J Med 369(2): 122-133. 17. Larkin, J., Chiarion-Sileni, V., Gonzalez, R., et al. Combined Nivolum$\mathrm{ab}$ and Ipilimumab or Monotherapy in Untreated Melanoma. (2015) N Eng J Med 373(1): 23-34.

18. Park, S.J., Cheong, H.I., Shin, J.I. Antibody depletion by bortezomib through blocking of antigen presentation. (2013) N Eng J Med 368(14): 1364-1365.

19. Chen, D.S., Mellman, I. Oncology meets immunology: the cancer-immunity cycle. (2013) Immunity 39(1): 1-10.

20. Wang, X.B., Fan, Z.Z., Anton, D., et al. CTLA4 is expressed on mature dendritic cells derived from human monocytes and influences their matura- tion and antigen presentation. (2011) BMC Immunology 12:21.

21. Tumeh, P.C., Harview, C.L., Yearley, J.H., et al. PD-1 blockade induces responses by inhibiting adaptive immune resistance. (2014) Nature 515: 568-571.

22. Ji, R.R., Chasalow, S.D., Wang, L., et al. An immune-active tumor microenvironment favors clinical response to ipilimumab. (2012) Cancer Immunol Immunother 61(7): 1019-1031.

23. Herbst, R.S., Soria, J.C., Kowanetz, M., et al. Predictive correlates of response to the anti-PD-L1 antibody MPDL3280A in cancer patients. (2014) Nature 515(7528): 563-567.

24. Gajewski, T.F., Schreiber, H., Fu, Y.X. Innate and adaptive immune cells in the tumor microenvironment. (2013) Nat Immunol 14(10): 1014-1022.

25. Grivennikov, S.I., Greten, F.R., Karin, M. Immunity, Inflammation, and Cancer. (2010) Cell 140(6): 883-899.

26. Landsberg, J., Kohlmeyer, J., Renn, M., et al. Melanomas resist T-cell therapy through inflammation-induced reversible dedifferentiation. (2012) Nature 490(7420): 412-416.

27. Coussens, L.M., Werb, Z. Inflammation and cancer. (2002) Nature 420(6917): 860-867.

28. Shacter, E., Weitzman, S.A. Chronic Inflammation and Cancer. (2002) Oncology 16(2): 217-226.

29. Hanahan, D., Weinberg, R.A. Hallmarks of cancer: the next generation. (2011) Cell 144(5): 646-674.

30. Ostrand-Rosenberg, S. Immune surveillance: a balance between protumor and antitumor immunity. (2008) Curr Opin Genet Dev 18(1): 11-18.

31. Lin, W-W., Karin, M. A-Cytokine-mediated link between innate immunity, inflammation, and cancer. (2007) J Clin Invest 117(5): 1175-1183.

32. Martel, C.D., Ferlay. J., Franceschi, S., et al. Global burden of cancers attributable to infections in 2008: a review and synthetic analysis. (2012) Lancet Oncology 13(6): 607-615.

33. Fuertes, M.B., Kacha, A.K., Kline, J., et al. Host type I IFN signals are required for antitumor $\mathrm{CD} 8+\mathrm{T}$ cell responses through $\mathrm{CD} 8\{$ alpha $\}+$ dendritic cells. (2011) J Exp Med 208(10): 2005-2016.

34. Gajewski, T.F., Fuertes, M.B., Woo, S.R. Innate immune sensing of cancer: clues from an identified role for type I IFNs. (2012) Cancer Immunol Immunother 61(8): 1343-1347.

35. Snyder, A., Makarov, V., Merghoub, T., et al. Genetic basis for clinical response to CTLA-4 blockade in melanoma. (2014) N Eng J Med 371(23): 2189-2199.

36. Gibson, S.J, Lindh, J.M., Riter, T.R., et al. Plasmacytoid dendritic cells produce cytokines and mature in response to the TLR7 agonists, imiquimod and resiquimod. (2002) Cell Immunol 218(1-2): 74-86.

37. Hemmi, H., Kaisho, T., Takeuchi, O., et al. Small anti-viral compounds activate immune cells via the TLR7 MyD88-dependent signaling pathway. (2002) Nat Immunol 3(2): 196-200.

38. Aspord, C., Tramcourt, L., Leloup, C., et al. Imiquimod inhibits melanoma development by promoting pDC cytotoxic functions and impeding tumor vascularization. (2014) J Invest Dermatol 134(10): 2551-2561.

39. Narayan, R., Nguyen, H., Bentow, J.J., et al. Immunomodulation by imiquimod in patients with high-risk primary melanoma. (2012) J Invest dermatol 132(1): 163-169.

40. Craft, N., Bruhn, K.W., Nguyen, B.D., et al. The TLR7 Agonist Imiquimod Enhances the Anti-Melanoma Effects of a Recombinant Listeria monocytogenes Vaccine. (2005) J Immunol 175(3): 1983-1990.

41. Ishikawa, H., Ma, Z., Barber, G.N. STING regulates intracellular DNA-mediated, type I interferon-dependent innate immunity. (2009) Nature 461(7265): 788-792

42. Wu, J., Sun, L., Chen, X., et al. Cyclic GMP-AMP is an endogenous second messenger in innate immune signaling by cytosolic DNA. (2013) Science 339(6121): 826-830.

43. Abe, T., Harashima, A., Xia, T., et al. STING recognition of cytoplasmic DNA instigates cellular defense. (2013) Mol cell 50(1): 5-15.

44. Sun, L., Wu, J., Du, F. Cyclic GMP-AMP synthase is a cytosolic DNA sensor that activates the type I interferon pathway. (2013) Science 339(6121): 786-791.

45. Woo, S.R., Fuertes, M.B., Corrales, L., et al. STING-dependent cytosolic DNA sensing mediates innate immune recognition of immunogenic tumors. (2014) Immunity 41(5): 830-842.

46. Ito, T., Amakawa, R., Inaba, M., et al. Differential Regulation of Human Blood Dendritic Cell Subsets by IFNs. (2001) J Immunol 166(5): 2961- 


\section{9.}

47. Corrales, L., Glickman, L.H., McWhirter, S.M., et al. Direct Activation of STING in the Tumor Microenvironment Leads to Potent and Systemic Tumor Regression and Immunity. (2015) Cell Rep 11(7): 1018-1030. 48. Spranger, S., Bao, R., Gajewski, T.F. Melanoma-intrinsic beta-catenin signalling prevents anti-tumor immunity. (2015) Nature 523(7559): 231235.

49. Yaguchi, T., Goto, Y., Kido, K., et al. Immune suppression and resistance mediated by constitutive activation of Wnt/beta-catenin signaling in human melanoma cells. (2012) J Immunol 189(5): 2110-2117.

50. Tago, K-i., Nakamura, T., Nishita, M., et al. Inhibition of Wnt signaling by ICAT, a novel -catenin-interacting protein. (2000) Genes Development 14: 1741-1749.

51. Jeong, Y.M., Li, H., Kim, S.Y., et al. Imidazole inhibits B16 melanoma cell migration via degradation of beta-catenin. (2010) J Pharm Pharmacol 62(4): 491-496.

52. Wilmott, J.S., Long, G.V., Howle, J.R., et al. Selective BRAF inhibitors induce marked T-cell infiltration into human metastatic melanoma. (2012) Clin Cancer Res 18(5): 1386-1394.

53. Frederick, D,T., Piris, A., Cogdill, A,P., et al. BRAF inhibition is associated with enhanced melanoma antigen expression and a more favorable tumor microenvironment in patients with metastatic melanoma. (2013) Clin Cancer Res 19(5): 1225-1231.

54. Boni, A., Cogdill, A.P., Dang, P., et al. Selective BRAFV600E inhibition enhances T-cell recognition of melanoma without affecting lymphocyte function. (2010) Cancer Res 70(13): 5213-5219.

55. Donia, M., Fagone, P., Nicoletti, F., et al. BRAF inhibition improves tumor recognition by the immune system: Potential implications for combinatorial therapies against melanoma involving adoptive T-cell transfer. (2012) OncoImmunology 1(9): 1476-1483.

56. Jiang, X., Zhou, J., Giobbie-Hurder, A., et al. The activation of MAPK in melanoma cells resistant to BRAF inhibition promotes PD-L1 expression that is reversible by MEK and PI3K inhibition. (2013) Clin Cancer Res 19(3): 598-609.

57. Khalili, J.S., Liu, S., Rodriguez-Cruz, T.G., et al. Oncogenic BRAF(V600E) promotes stromal cell-mediated immunosuppression via induction of interleukin-1 in melanoma. (2012) Clin Cancer Res 18(19): 5329-5340.

58. Cooper, Z.A., Juneja, V.R., Sage, P.T., et al. Response to BRAF inhibition in melanoma is enhanced when combined with immune checkpoint blockade. (2014) Cancer Immunol Res 2(7): 643-654.

59. Aris, M., Barrio, M.M. Combining immunotherapy with oncogene-targeted therapy: a new road for melanoma treatment. (2015) Fron Immunol 6: 46 .

60. Hu-Lieskovan, S., Mok, S., Homet Moreno, B., et al. Improved antitumor activity of immunotherapy. (2015) Sci Transl Med 7(279): 279.

61. Lugade, A.A., Sorensen, E.W., Gerber, S.A., et al. Radiation-Induced IFN- Production within the Tumor Microenvironment Influences Antitumor Immunity. (2008) J Immunol 180(5): 3132-3139.

62. Lugade, A.A., Moran, J.P., Gerber, S.A., et al. Local Radiation Therapy of B16 Melanoma Tumors Increases the Generation of Tumor Antigen-Specific Effector Cells That Traffic to the Tumor. (2005) J Immunol 174(12): 7516-7523.

63. Sharabi, A.B., Nirschl, C.J., Kochel, C.M., et al. Stereotactic Radiation Therapy Augments Antigen-Specific PD-1-Mediated Antitumor Immune Responses via Cross-Presentation of Tumor Antigen. (2015) Cancer immunol Res 3(4): 345-355.

64. Reits, E.A., Hodge, J.W., Herberts, C.A., et al. Radiation modulates the peptide repertoire, enhances MHC class I expression, and induces successful antitumor immunotherapy. (2006) J Exp Med 203(5): 1259-1271.

65. Twyman-Saint Victor, C., Rech, A.J., Maity, A., et al. Radiation and dual checkpoint blockade activate non-redundant immune mechanisms in cancer. (2015) Nature 520(7547): 373-377.

66. Spranger, S., Koblish, HK., Horton, B., et al. Mechanism of tumor rejection with doublets of CTLA-4, PD-1/PD-L1, or IDO blockade involves restored IL-2 production and proliferation of $\mathrm{CD} 8(+) \mathrm{T}$ cells directly within the tumor microenvironment. ( 2014) J Immunother Cancer 2: 3.

67. Spranger, S., Spaapen, R.M., Zha, Y., et al. Up-regulation of PD-L1, IDO, and $\mathrm{T}_{\text {regs }}$ in the melanoma tumor microenvironment is driven by CD8+ T cells. (2013) Sci transl Med 5(200): 200ra116.

68. Chen, W., Liang, X., Peterson, A.J., et al. The Indoleamine 2,3-Dioxygenase Pathway Is Essential for Human Plasmacytoid Dendritic Cell-Induced Adaptive T Regulatory Cell Generation. (2008) J Immunol 181(8): 5396-5404.

69. Holmgaard, R.B., Zamarin, D., Munn, D.H., et al. Indoleamine 2,3-dioxygenase is a critical resistance mechanism in antitumor $\mathrm{T}$ cell immunotherapy targeting CTLA-4. (2013) J Exp Med 210(7): 1389-402.

70. Takamatsu, M., Hirata, A., Ohtaki, H., et al. Inhibition of indoleamine 2,3-dioxygenase 1 expression alters immune response in colon tumor microenvironment in mice. (2015) Cancer Sci 106(8): 1008-1015.

71. Uyttenhove, C., Pilotte, L., Theate, I., et al. Evidence for a tumoral immune resistance mechanism based on tryptophan degradation by indoleamine 2,3-dioxygenase. (2003) Nat Med 9(10): 1269-1274.

72. Chiba, S., Baghdadi, M., Akiba, H., et al. Tumor-infiltrating DCs suppress nucleic acid-mediated innate immune responses through interactions between the receptor TIM-3 and the alarmin HMGB1. (2012) Nat Immunol 13(9): 832-842.

73. Sabatos, C.A., Chakravarti, S., Cha, E., et al. Interaction of Tim-3 and Tim-3 ligand regulates Thelper type 1 responses and induction of peripheral tolerance. (2003) Nat Immunol 4(11): 1102-1110.

74. Sakuishi, K., Ngiow, S.F., Sullivan, J.M., et al. TIM3+FOXP3+ regulatory $\mathrm{T}$ cells are tissue-specific promoters of T-cell dysfunction in cancer. (2013) OncoImmunology 2(4): e23849.

75. Sakuishi, K., Apetoh, L., Sullivan, J.M., et al. Targeting Tim-3 and PD-1 pathways to reverse $\mathrm{T}$ cell exhaustion and restore anti-tumor immunity. (2010) J Exp Med 207(10): 2187-2194.

76. Fourcade, J., Sun, Z., Benallaoua, M., et al. Upregulation of Tim-3 and PD-1 expression is associated with tumor antigen-specific CD8+ T cell dysfunction in melanoma patients. (2010) J Exp Med 207(10): 2175-2186.

77. Huang, C.T., Workman, C.J., Flies, D., et al. Role of LAG-3 in regulatory $\mathrm{T}$ cells. (2004) Immunity 21(4): 503-513.

78. Workman, C.J., Vignali, D.A. Negative Regulation of T Cell Homeostasis by Lymphocyte Activation Gene-3 (CD223). (2005) J Immunol 174(2): 688-695.

79. Grosso, J.F., Kelleher, C.C., Harris, T.J., et al. LAG-3 regulates CD8+ T cell accumulation and effector function in murine self- and tumor-tolerance systems. (2007) J Clin Invest 117(11): 3383-3392.

80. Woo, S.R., Turnis, M.E., Goldberg, M.V., et al. Immune inhibitory molecules LAG-3 and PD-1 synergistically regulate T-cell function to promote tumoral immune escape. (2012) Cancer Res 72(4): 917-927.

81. Casati, C., Camisaschi, C., Rini, F., et al. Soluble human LAG-3 molecule amplifies the in vitro generation of type 1 tumor-specific immunity. (2006) Cancer Res 66(8): 4450-4460.

82. Brignone, C., Escudier. B., Grygar, C., et al. A phase I pharmacokinetic and biological correlative study of IMP321, a novel MHC class II agonist, in patients with advanced renal cell carcinoma. (2009) Clin Cancer Res 15(19): 6225-6231.

83. Brignone, C., Escudier, B., Grygar, C.,et al. First-line chemoimmunotherapy in metastatic breast carcinoma: combination of paclitaxel and IMP321 (LAG-3Ig) enhances immune responses and antitumor activity. (2010) J Transl Med 8: 71.
Online ISSN: 2381-0858

Journal Title: Investigative Dermatology and Venereology Research Journal Short Name: Invest Dermatol Venereol Res
Ommega Online Publishers

E-mail: editor.dermatology@ommegaonline.org

Website: www.ommegaonline.org 